Louisiana State University

LSU Digital Commons

Faculty Publications

Department of Biological Sciences

$1-10-2013$

\title{
Hijacking membrane transporters for arsenic phytoextraction
}

Melissa S. LeBlanc

University of Georgia

Elizabeth C. McKinney

University of Georgia

Richard B. Meagher

University of Georgia

Aaron P. Smith

Louisiana State University

Follow this and additional works at: https://digitalcommons.Isu.edu/biosci_pubs

\section{Recommended Citation}

LeBlanc, M., McKinney, E., Meagher, R., \& Smith, A. (2013). Hijacking membrane transporters for arsenic phytoextraction. Journal of Biotechnology, 163 (1), 1-9. https://doi.org/10.1016/j.jbiotec.2012.10.013

This Article is brought to you for free and open access by the Department of Biological Sciences at LSU Digital Commons. It has been accepted for inclusion in Faculty Publications by an authorized administrator of LSU Digital Commons. For more information, please contact ir@lsu.edu. 


\title{
Hijacking membrane transporters for arsenic phytoextraction
}

\author{
Melissa S. LeBlanc ${ }^{a}$, Elizabeth C. McKinney ${ }^{a}$, Richard B. Meaghera, and Aaron P. Smith ${ }^{\mathrm{b},{ }^{*}}$ \\ ${ }^{a}$ Genetics Department, University of Georgia, Athens, GA 30602 \\ ${ }^{b}$ Department of Biological Sciences, Louisiana State University, Baton Rouge, LA 70803
}

\begin{abstract}
Arsenic is a toxic metalloid and recognized carcinogen. Arsenate and arsenite are the most common arsenic species available for uptake by plants. As an inorganic phosphate (Pi) analog, arsenate is acquired by plant roots through endogenous Pi transport systems. Inside the cell, arsenate is reduced to the thiol-reactive form arsenite. Glutathione (GSH)-conjugates of arsenite may be extruded from the cell or sequestered in vacuoles by members of the ATP-binding cassette $(\mathrm{ABC})$ family of transporters. In the present study we sought to enhance both plant arsenic uptake through Pi transporter overexpression, and plant arsenic tolerance through $\mathrm{ABC}$ transporter overexpression. We demonstrate that Arabidopsis thaliana plants overexpressing the high-affinity Pi transporter family members, AtPht1;1 or AtPht1;7, are hypersensitive to arsenate due to increased arsenate uptake. These plants do not exhibit increased sensitivity to arsenite. Cooverexpression of the yeast $\mathrm{ABC}$ transporter $Y C F 1$ in combination with AtPht1;1 or AtPht1;7 suppresses the arsenate-sensitive phenotype while further enhancing arsenic uptake. Taken together, our results support an arsenic transport mechanism in which arsenate uptake is increased through Pi transporter overexpression, and arsenic tolerance is enhanced through YCF1-mediated vacuolar sequestration. This work substantiates the viability of coupling enhanced uptake and vacuolar sequestration as a means for developing a prototypical engineered arsenic hyperaccumulator.
\end{abstract}

\section{Keywords}

arsenate; phytoremediation; yeast cadmium factor 1; phosphate transporter; glutathione; $\mathrm{ABC}$ transporter

\section{Introduction}

Long-term human exposure to arsenic has been linked to an increased incidence of several cancers and non-cancer skin pathologies (Akter et al., 2005; Duker et al., 2005; Smith et al., 1992). Unfortunately, environmental levels of arsenic exceeding safe standards set by the WHO and EPA are found at many locations worldwide [e.g. India (Patel et al., 2005), Bangladesh (Smith et al., 2000), China (Xia and Liu, 2004), Vietnam (Berg et al., 2001), Australia (Smith et al., 2003), and the U.S. (Durant et al., 2004; Erickson and Barnes, 2005; Welch et al., 2000)]. Concerned over the health issues associated with arsenic pollution, we

(C) 2012 Elsevier B.V. All rights reserved.

Tel.: +225-578-7643; fax: 225-578-2597, apsmith@1su.edu.

Publisher's Disclaimer: This is a PDF file of an unedited manuscript that has been accepted for publication. As a service to our customers we are providing this early version of the manuscript. The manuscript will undergo copyediting, typesetting, and review of the resulting proof before it is published in its final citable form. Please note that during the production process errors may be discovered which could affect the content, and all legal disclaimers that apply to the journal pertain. 
seek to develop strategies for extracting arsenic from contaminated water and soil (Meagher et al., 2007).

Arsenic is present in the majority of aerobic soils as the toxic oxyanion arsenate $\left(\mathrm{AsO}_{4}{ }^{-3}\right)$. Due to its chemical similarity to inorganic phosphate $\left(\mathrm{Pi} ; \mathrm{PO}_{4}{ }^{-3}\right)$, arsenate gains access to plant cells by hijacking endogenous Pi transport systems (Abedin et al., 2002; Meharg and Macnair, 1990; Wang et al., 2002). Due to the essential roles Pi plays in growth and development, plants have evolved a variety of responses to facilitate uptake under Pilimiting conditions (Raghothama, 1999; Yang and Finnegan, 2010). One such response is the induction of high-affinity Pi transporter expression (Muchhal et al., 1996; Shin et al., 2004). The mobilization of phosphorus from mature shoot tissues to actively growing tissues is another Pi starvation response (Jeschke et al., 1997; Mudge et al., 2002; Nagarajan et al., 2011), which may be achieved in part through regulation of Pi transporter expression. The Arabidopsis genome encodes nine high-affinity Pi transporters (Pht1;1 - Pht1;9, hereafter called PHT1 - PHT9), eight of which are induced in roots under Pi-deficient conditions (PHT1-5 and PHT7-9, Mudge et al., 2002). While the relative contribution of the nine Arabidopsis PHT family members to arsenic uptake is not yet known, PHT1 and PHT4 have both been implicated in arsenate transport. pht 1 null mutants are moderately arsenate tolerant, while pht 1 pht 4 double mutants are significantly arsenate tolerant, indicating that arsenic uptake is compromised by mutations in these Pi transporters (Shin et al., 2004). Plants overexpressing PHT1, on the other hand, have been shown to be arsenate-sensitive (Catarecha et al., 2007). In the present study, we sought to increase plant arsenic uptake through overexpression of the high-affinity Arabidopsis Pi transporters PHT1 (PHT1ox) and PHT7 (PHT7ox).

Several members of the ATP-binding cassette (ABC) transporter family have been implicated in the transport of arsenic across membranes. For example, HsABCC1/HsMRP1 over-expression has been linked to arsenic resistance in a tumor-derived cell line (Vernhet et al., 1999). Furthermore, HsABCC1 homologs contribute to arsenic detoxification in the nematode Caenorhabditis elegans (Broeks et al., 1996; Schwartz et al., 2010), the protozoan Leishmania tarentolae (Papadopoulou et al., 1994), and the yeast Saccharomyces cerevisiae (Song et al., 2003; Guo et al., 2012). Whereas HsABCC1 is found at the plasma membrane, the ABC transporter Yeast Cadmium Factor 1 (YCF1) has been localized to the vacuolar membrane in both yeast (Wemmie and Moye-Rowley, 1997) and transgenic Arabidopsis plants (Song et al., 2003). Several YCF1 homologs in plants have been found at the tonoplast as well (Jaquinod et al., 2007; Klein et al., 2006; Liu et al., 2001). More recently, two transporters in Arabidopsis, AtABCC1 and AtABCC2, were shown to contribute to arsenic tolerance via vacuolar sequestration of As(III)-phytochelatin conjugates (Song et al., 2010). While plasma membrane ABC transporters are involved in substrate extrusion, vacuolar $\mathrm{ABC}$ transporters are involved in substrate sequestration. Although both cellular extrusion and vacuolar sequestration of arsenic are viable mechanisms for arsenic detoxification, only vacuolar sequestration would result in the combination of increased arsenic resistance and accumulation. In the present study we sought to increase plant arsenic resistance through overexpression of yeast YCF1 (YCF1ox).

Coupling two intuitively mutually exclusive activities - increased arsenic uptake and increased arsenic tolerance - is essential to engineering a plant appropriate for arsenic phytoremediation. Toward this end, we created transgenic Arabidopsis plants overexpressing Arabidopsis Pi transporters (PHT1ox or PHT7ox) either alone or in combination with YCF1ox. Analysis of the transgenic plants revealed that overexpression of PHT1, PHT7, or YCF1 enhanced plant arsenic accumulation. Furthermore, coupling PHTand $Y C F 1$-overexpression conferred both enhanced arsenic tolerance and accumulation on transgenic plants. Our results support the viability of coupling enhanced uptake with 
enhanced vacuolar sequestration as a means for developing a prototypical engineered arsenic hyperaccumulator.

\section{Materials and methods}

\subsection{Plant growth and treatments}

For all experiments, wild-type Arabidopsis thaliana (ecotype Columbia) and transgenic seeds were surface-sterilized and grown under a long-day photoperiod ( $16 \mathrm{~h}$ light $/ 8 \mathrm{~h}$ dark) at $22^{\circ} \mathrm{C}$ with an average light intensity of $60 \mu \mathrm{mol} \mathrm{m}^{-2} \mathrm{~s}^{-1}$. Seedlings were grown on plates containing half-strength Murashige and Skoog (MS) salts (Murashige and Skoog, 1962) with $1 \%(\mathrm{wt} / \mathrm{vol}$ ) sucrose (MS plates) for the duration indicated. For assaying arsenate exposure phenotypes and quantifying transgene abundance, plants were grown on MS plates supplemented with $1.2 \%$ (vertically-oriented) or $1.0 \%$ (horizontally-oriented) agar (Caisson). Otherwise, MS media was solidified with agar (Type E, Sigma) concentrations of $0.5 \%$ for horizontally oriented plates and $0.9 \%$ for vertically oriented plates. To generate Pistarved tissue for $P H T 1$ and $P H T 7 \mathrm{cDNA}$ amplification, seedlings were grown in liquid MS media on a rotary shaker for $10 \mathrm{~d}$ and subsequently transferred to MS media lacking Pi for an additional $2 \mathrm{~d}$. For seedling treatments, sodium arsenite, sodium arsenate, or buthionine sulfoximine (Sigma) was added to plant media after autoclaving at the indicated concentration from an aqueous, sterile stock solution.

\subsection{Reverse Transcription-PCR}

RNA was isolated using the Spectrum Plant Total RNA Kit (Sigma), except for transgene quantification experiments, which utilized the Qiagen RNeasy plant mini kit (Qiagen). One microgram of RNA was DNAse treated using RQ1 RNAse-free DNAse (Promega), and complementary DNA synthesis was performed with an oligo $(\mathrm{dT})_{20}$ primer using the Superscript III First-Strand Synthesis System for RT-PCR (Invitrogen). Twenty $\mu \mathrm{L}$ reaction volumes containing $2 \mu \mathrm{M}$ each of sense and antisense primers, $4 \mu \mathrm{L}$ of 20 -fold diluted cDNA, and $10 \mu \mathrm{L}$ SYBR Green PCR Master Mix (Applied Biosystems) were used for quantitative RT-PCR (qRT-PCR) experiments using an Applied Biosystems 7500 RealTime PCR instrument. The ACTIN2 transcript was used as the endogenous control, and relative expression levels for each Arabidopsis Pi transporter were calculated based on four to six replicates using the $2^{-\Delta \Delta \mathrm{Ct}}$ method of relative quantification (Livak and Schmittgen, 2001). These data were normalized to transcript abundance in untreated control tissues. The transcript abundance of $Y C F 1, P H T 1$ or $P H T 7$ in overexpression lines relative to wild-type plants was expressed as $2^{-\Delta \mathrm{Ct}}$ rather than $2^{-\Delta \Delta \mathrm{Ct}}$ since the $Y C F 1$ transcript is not detected in wild-type Arabidopsis.

\subsection{Cloning and expression of YCF1, PHT1, and PHT7 in plants}

The 4548 nucleotide $Y C F 1$ coding sequence was amplified in two pieces from genomic DNA isolated from the BY4742 S. cerevisiae parental strain produced by the Saccharomyces Genome Deletion Project (http://www-sequence.stanford.edu/group/ yeast_deletion_project/deletions3.html) and assembled using overlap extension PCR (Ho et al., 1989). The $5^{\prime}$ half of $Y C F 1(2.6 \mathrm{~Kb})$ was modified during PCR to eliminate an internal $\mathrm{NcoI}$ site, using the primers YCF1-XhNcIS, YCF1-NcMutN, YCF1-NcMutS, and YCF1PstIN (all primer sequences are shown in Table S1). This $2.6 \mathrm{~Kb}$ fragment was cloned into pBluescript KS via the $X h o I$ and Pst restriction sites ( $p B S:: Y C F 1-2.6)$. The $3^{\prime}$ half of $Y C F 1(2.0 \mathrm{~Kb})$ was amplified using the primers YCF1-PstIS and YCF1-ScBmN, and cloned into $p B S:: Y C F 1-2.6$ using the PsI and BamHI restriction sites ( $p B S:: Y C F 1)$. The $N c o I$ and $B a m H I$ restriction sites flanking $Y C F 1$ were used to subclone the $Y C F 1$ coding sequence into a constitutive vegetative $A C T I N 2$ promoter/terminator cassette ( $A 2 p t)$ (described in Kandasamy et al., 2002), within the binary vector pCambia-1300 (pCambia::A2pt::YCF1). 
The $P H T 1$ and $P H T 7 \mathrm{cDNAs}$ were amplified from total RNA extracted from 12-day-old wild-type Arabidopsis seedlings that had been starved for Pi for two days. The PHT1 sequence was modified using overlap extension PCR (Ho et al., 1989) to eliminate an internal NcoI site, with the primers PHT1_S1, PHT1_A894, PHT1_S863, and PHT1_A1575noHA. A single set of flanking primers was used to amplify the constructed sequence (PHT1_flank_S and PHT1_flank_A) prior to cloning into an ACTIN2 promoter/ terminator cassette (Kandasamy et al., 2002) via $\mathrm{NcoI}$ and HindIII restriction sites within the binary vector pCambia-1300 (pCambia::A2pt::PHT1). The PHT7 sequence was amplified in 3 pieces and modified using overlap extension PCR (Ho et al., 1989) to eliminate internal $B s p \mathrm{HI}$ and $K p n I$ restriction sites. A second $K p n I$ site was eliminated via point mutation in the PHT7_S1 primer. In addition, a serine codon was inserted following the methionine start to maintain in-frame cloning via a $\mathrm{NcoI}$ restriction site. The primers used for amplification were: PHT7_S1, PHT7_A876, PHT7_S845, PHT7_A1073, PHT7_S1045, and PHT7_A1608noHA. A single set of flanking primers was used to amplify the constructed sequence (PHT7_flank_S and PHT7_flank_A) prior to cloning into the ACTIN2 promoter/ terminator cassette via $\mathrm{NcoI} / \mathrm{BspHI}$ and SalI restriction sites within the binary vector pCambia-1300 (pCambia::A2pt::PHT7).

Wild-type Arabidopsis plants were transformed independently with each membrane transporter overexpression construct using Agrobacterium-mediated transformation (Clough and Bent, 1998). PCR analysis of genomic DNA from hygromycin-resistant plants using one vector-specific and one gene-specific primer was used to verify the presence of the correct transgene within each plant line. The segregation of hygromycin resistance was used to identify T3 plant lines homozygous for each construct. YCF1 and PHT1 or PHT7 cooverexpression plants were generated through cross-fertilization between appropriate transgenic plants.

\subsection{Arsenic accumulation}

Plant samples were swirled for $2 \mathrm{~min}$ in $0.01 \mathrm{M} \mathrm{HCl}$, followed by two rinses in deionized water to remove residual arsenic-containing media from tissue surfaces (Aldrich et al., 2003). The samples were prepared for elemental analysis based on methods described in $\mathrm{Li}$ et al., 2004. Samples were dried for $3 \mathrm{~d}$ at $60^{\circ} \mathrm{C}$. Prior to arsenic analysis, dried plant tissue was digested overnight with 7 parts nitric acid : 1 part perchloric acid, diluted with water, and passed through a 0.45 micron syringe filter unit. Arsenic levels in these samples were determined using ICP-OES (Chemical Analysis Laboratory, Center for Applied Isotope Studies, University of Georgia).

\subsection{Glutathione depletion experiments}

Seedlings were germinated on MS plates, oriented vertically. 5-day old seedlings were transferred to a new MS plate supplemented with $250 \mu \mathrm{M}$ BSO or $100 \mu \mathrm{M}$ sodium arsenate, or both chemicals. At the time of transfer, root tips were marked with a dot on the bottom of the Petri plate. Following three additional days of growth, Image $\mathrm{J}$ was used to measure the length of root growth in pixels from the dot to the new location of the root tip (Abramoff et al., 2004).

\subsection{Quantification of thiol content}

Sulfhydryl groups were quantified in seedlings exposed to BSO, arsenate, or both (as described above), using Ellman's Reagent [DTNB, (Ellman, 1959), Pierce Protein Research Products, Thermo Fisher Scientific] based on a method modified from Lee et al (2003). Approximately $100 \mathrm{mg}$ of plant tissue was ground in liquid nitrogen and resuspended in 300 $\mu \mathrm{L} 1 \mathrm{M} \mathrm{NaOH}$ and $1 \mathrm{mg} / \mathrm{L} \mathrm{NaBH}_{4}$. After a $5 \mathrm{~min}$ spin at $13,000 \mathrm{x}$ g at $4^{\circ} \mathrm{C}$, the supernatant was moved to a new tube, and $50 \mu \mathrm{L}$ of concentrated $\mathrm{HCl}$ was added. Samples were 
vortexed briefly and spun at $13,000 \mathrm{x}$ g for $5 \mathrm{~min}$ at room temperature. Eighty $\mu \mathrm{L}$ of supernatant was transferred to a new tube containing $1 \mathrm{~mL}$ of reaction buffer $(0.1 \mathrm{M}$ sodium Pi pH 8.0, containing $5 \mathrm{mM}$ EDTA) and $20 \mu \mathrm{L}$ of $4 \mathrm{mg} / \mathrm{mL}$ Ellman's reagent. Samples were incubated for $15 \mathrm{~min}$ at $30^{\circ} \mathrm{C}$, at which time the absorbance at $\mathrm{A}_{412} \mathrm{~nm}$ was recorded relative to blanked control with reaction buffer.

\subsection{Statistics}

Where indicated, statistical significance was determined using Student's t-Test $(\mathrm{p}<0.05)$, and standard deviations or standard errors are shown in the bar graphs.

\section{Results}

\subsection{Selection of Pi transporters for overexpression}

We assayed the transcript abundance of the Arabidopsis high-affinity Pi transporter (PHT) family in shoot and root tissues of arsenic-exposed seedlings. Observing increased $P H T$ transcript abundance in response to arsenate may deem one or more PHT proteins worthy of targeting for enhancing arsenate acquisition. However, no $P H T$ transcripts were induced in shoots or roots of plants exposed to $150 \mu \mathrm{M}$ sodium arsenate for 21 days, but rather, PHT8 transcript abundance decreased 5.6-fold in roots (Fig. 1; PHT3 and PHT6 transcripts were consistently below the limit of detection in our assays). Since arsenate exposure did not implicate any PHTs in arsenate acquisition from the substrate by roots, we selected one vegetative (PHTI) and one reproductive (PHT7) Pi transporter for constitutive overexpression in wild-type Arabidopsis plants, based on their contrasting root and floral RNA expression levels, respectively (Mudge et al., 2002; Fig. 2).

\subsection{Generation of transgenic Arabidopsis overexpressing PHT and/or YCF1}

We cloned the coding sequences of each of PHT1, PHT7, and YCF1 into binary vectors for plant expression, under the control of the constitutive, vegetative ACTIN2 promoter and terminator sequences (A2pt; Kandasamy et al., 2002). Agrobacterium-mediated transformation was used to independently introduce the A2pt::PHT1 (PHT1ox), A2pt::PHT7 (PHT7ox), and A2pt::YCF1 (YCF1ox) constructs into wild-type Arabidopsis. Plant lines homozygous for a single insertion of each transgene were identified by hygromycin resistance of the T3 generation, and used to generate PHT1ox YCF1ox and PHT7ox YCF1ox co-overexpressing plant lines through crossing. Overexpression of the YCF1, PHT1, and $P H T 7$ transgenes in the lines selected for further study were verified using quantitative Reverse Transcription-Polymerase Chain Reaction (qRT-PCR; Fig. 3). Transcript levels from each transgene were lower in the co-overexpression lines as compared to the parental, single transformants, although expression of the endogenous $A C T 2$ gene was unaffected, as determined via qRT-PCR analysis (data not shown). This could be the result of partial silencing of the $A C T 2$ promoters driving transgene expression, but not of the endogenous ACT2 promoter, through small RNA-dependent promoter methylation (Eamens et al., 2008). Indeed, endogenous promoters appear less sensitive to this co-suppression silencing mechanism than transgene promoters (Okano et al., 2008).

\subsection{Growth phenotypes of transgenic lines on arsenate}

Initial screening of segregating YCF1ox lines in the presence of arsenate $(200 \mu \mathrm{M})$ demonstrated that $Y C F 1$-overexpression conferred arsenate tolerance on 30-day-old Arabidopsis seedlings (Fig. 4A), presumably due to vacuolar sequestration of arsenic via the transport activity of YCF1. In contrast, assaying the growth of several independent PHT1ox and PHT7ox lines in the presence of arsenate $(150 \mu \mathrm{M})$ demonstrated that PHToverexpression conferred arsenate sensitivity on 30-day-old Arabidopsis seedlings (Fig. 4B), presumably due to elevated arsenate uptake. YCF1ox and PHTox co-overexpression lines 
were generated based on the hypothesis that the in planta activities of these transporters would complement each another. Indeed, overexpression of the $Y C F 1$ transgene in a PHToX background rescued the arsenate-sensitivity of the PHTox lines (Fig. 4B). Based on visual screening for the best growth on arsenate, lines A2pt::PHT1-16 x A2pt::YCF1-16 (PHT1ox YCF1ox) and A2pt::PHT7-8 X A2pt::YCF1-16(PHT7ox YCF1ox) were selected for further study (Fig. 4A,B). Whole-seedling fresh weights of 30-day-old plants grown on $150 \mu \mathrm{M}$ arsenate were measured to quantify the arsenate tolerance/sensitivity of the various transgenic lines. Both PHTox YCF1ox co-expression lines accumulated at least 2.5 -fold more biomass than the wild type, and approximately 4-fold more biomass than each corresponding single PHTox-expressor (Fig. 4C). Separating root and shoot fresh weights indicated that root growth in the co-expressors is enhanced to a greater extent than shoot growth relative to wild type (Supplementary material, Fig. S1). This may be linked to a change in root morphology in plants containing a PHT1ox or PHT7ox transgene (Supplementary material, Fig. S2), which could be the result of altered Pi uptake/ homeostasis and/or increased arsenate accumulation (see below). Notably, relative to wildtype plants, PHT1ox and PHT7ox plants were not hypersensitive to arsenite $\left(\mathrm{AsO}_{3}{ }^{-3}\right)$, the electrochemically reduced form of arsenate (Supplementary material, Fig. S3).

\subsection{Arsenic accumulation of the transgenic lines}

The arsenic content of seedlings grown in the presence of $150 \mu \mathrm{M}$ sodium arsenate for 21 days was measured using Inductively Coupled Plasma - Optical Emission Spectrometry (ICP-OES; Fig. 5). Both PHT1ox and PHT7ox plants accumulated significantly higher arsenic levels than wild-type plants $(\mathrm{p}<0.05)$, consistent with increased arsenate acquisition in these lines. Similarly, YCF1ox plants contained significantly higher As levels than wild type ( $\mathrm{p}<0.05$ ), consistent with previously reported vacuolar metal-sequestering activities of YCF1 in yeast cells (Ghosh et al., 1999) and transgenic Arabidopsis (Guo et al., 2012). Whereas YCF1ox, PHT1ox, and PHT7ox lines contained 125\% to 135\% higher arsenic levels relative to wild type, $P H T O X Y C F 10 x$ co-expressors accumulated significantly higher arsenic levels $(\sim 150 \%$; $\mathrm{p}<0.05)$.

\subsection{Glutathione depletion differentially affects the arsenate-sensitivity of the transgenic lines}

Arsenate exposure induces glutathione (GSH) synthesis in plants, and in so doing, increases in planta sulfhydryl levels (Li et al., 2004; Schat et al., 2002; Srivastava et al., 2009). The synthetic amino acid buthionine sulfoximine (BSO), on the other hand, inhibits GSH synthesis (Griffith and Meister, 1979), depleting in planta sulfhydryl levels. We quantified the effect of GSH depletion by BSO treatment on root arsenate-sensitivity of PHToX, YCF1ox, and PHTox YCF1ox plants. Five-day old seedlings were exposed to $250 \mu \mathrm{M}$ BSO, $100 \mu \mathrm{M}$ arsenate, or both chemicals for 3 days, and the change in primary root elongation was measured (Fig. 6A). The root growth of transgenic plants was not significantly altered by BSO, relative to wild-type plants ( $\mathrm{p}<0.05$ ). In contrast, root growth of all transgenic lines was more sensitive to arsenate compared to wild type $(\mathrm{p}<0.05)$, which may reflect the increased arsenate accumulation in these lines (Fig. 5). This was surprising based on our data from 30-day-old plants grown on $150 \mu \mathrm{M}$ arsenate, in which the PHTox YCF1ox coexpressors exhibited increased root biomass relative to wild type (Supplementary material, Fig. S1). This apparent discrepancy likely reflects a difference in root morphology (Supplementary material, Fig. S2; see Discussion). Root growth of PHTox seedlings was more sensitive to arsenate or the combination of arsenate and BSO than YCF1ox seedlings (p < 0.05; Fig. 6A). Also, depletion of GSH magnified root arsenate sensitivity in all PHToX and PHTox YCF1ox lines, as compared to YCF1ox, with the PHTox YCF1ox co-expressors being less sensitive than lines overexpressing only PHT1 or $P H T 7$ (p < 0.05; Fig. 6A). Together these results highlight the importance of GSH in compensating for the increased 
cytotoxic effects of elevated arsenic uptake in the PHTox and PHTox YCF1ox lines, and that although GSH is also required for the arsenic-transporting activity of YCF1, the level of GSH depletion achieved by $250 \mu \mathrm{M}$ BSO exposure was not enough to eliminate the beneficial effect of $Y C F 1$ overexpression. However, increasing the concentrations of arsenate and BSO to $150 \mu \mathrm{M}$ and $500 \mu \mathrm{M}$, respectively, did eliminate the beneficial role of YCF1 (Supplementary material, Fig. S4).

To further characterize the role of GSH and other thiols in the arsenate-responses of the transgenics, we quantified free sulfhydryl levels in all genotypes exposed to $250 \mu \mathrm{M} \mathrm{BSO}$, $100 \mu \mathrm{M}$ arsenate, or both chemicals, using Ellman's reagent (Fig. 6B). As expected, all genotypes contained significantly elevated free sulfhydryl levels in the presence of $100 \mu \mathrm{M}$ arsenate relative to exposure to $250 \mu \mathrm{M}$ BSO ( $<<0.05$ ). Intermediate free sulfhydryl levels were observed in all genotypes when plants were treated with both $100 \mu \mathrm{M}$ arsenate and 250 $\mu \mathrm{M}$ BSO. This result is consistent with the contrasting effects of arsenate [stimulatory ( $\mathrm{Li}$ et al., 2006)] and BSO [inhibitory (Griffith and Meister, 1979)] on cellular thiol peptide levels. Notably, thiol peptide levels of YCF1ox plants were comparable to those of wild-type plants in the presence of arsenate. In contrast, the PHTox YCFlox plants contained higher free sulfhydryl levels in the presence of arsenate than plants overexpressing $Y C F 1$ alone, but did not have elevated thiol levels relative to PHTox plants in the presence of BSO and arsenate ( $\mathrm{p}<0.05$; Fig. 6B). Thus, the enhanced root growth seen in the PHTox YCF1ox lines grown under these conditions, relative to PHTox lines, (Fig. 6A) is not linked to a direct de novo increase in free sulfhydryl levels, but is likely due to enhanced vacuolar sequestration activity of YCF1.

\section{Discussion}

The form of phosphorus available to plant roots for uptake and assimilation is inorganic phosphate $(\mathrm{Pi})$. Unfortunately, one of the most common environmental forms of the toxic metalloid arsenic is the $\mathrm{Pi}$ analog, arsenate. Due to the chemical similarity between Pi and arsenate, arsenate is absorbed by plant roots through the Pi transport system (Abedin et al., 2002; Meharg and Macnair, 1990; Wang et al., 2002). Hence, suppression of high-affinity Pi transport systems seems to be a common mechanism for acquired arsenic tolerance (Meharg and Macnair, 1992). This is consistent with our observation of decreased PHT8 transcripts in roots of arsenate-treated plants (Fig. 1). Similarly, Catarecha et al (2007) reported repression of the Pi transporter PHT1 in Pi-starved plants (3d) exposed to arsenate (8h).

Because arsenate has been shown to enter plants via the Pi transport system, and pht 1 pht 4 double mutants in Arabidopsis exhibit increased tolerance to arsenate (Shin et al., 2004), we reasoned that over-expression of high-affinity Pi transporters in Arabidopsis would lead to plants with increased arsenate uptake. Based on the tissue localization of members of the Arabidopsis $P H T$ family (Mudge et al., 2002), we chose one vegetatively expressed (PHTI) and one reproductively expressed (PHT7) Pi transporter for our studies. Our qRT-PCR studies on seedlings grown under Pi-replete conditions confirmed the vegetative expression previously seen for $P H T 1$ and floral expression for $P H T 7$ (Fig. 2). We chose to include both a vegetative and a reproductive PHT in our study in case either of these two classes of Pi transporters has evolved an ability to discriminate between arsenate and Pi.

Catarecha et al (2007) demonstrated that overexpression of PHT1 (35S::PHTI) conferred increased arsenate sensitivity on plants. Herein, we showed that overexpression of the $\mathrm{Pi}$ transporters PHT1 (A2pt::PHT1; PHT1ox) or PHT7 (A2pt::PHT7; PHT7ox) conferred arsenate sensitivity (Fig. 4), as well as enhanced arsenic accumulation (Fig. 5). We also demonstrated that the PHTox plants are not sensitive to the closely related, but chemically reduced oxyanion, arsenite (Supplementary material, Fig. S3). This result is consistent with 
previously published reports that arsenite is not taken up through the Pi transport system in plants (Abedin et al., 2002; Bienert et al., 2008; Isayenkov and Maathuis, 2008). Under our conditions, PHT7ox plants appeared marginally more sensitive to arsenate than PHT1ox plants (Supplementary material, Fig. S1, Fig. 4, and Fig. 6A), but accumulated about the same quantity of arsenic (Fig. 5). Since PHT7 transcript abundance was greater in PHT7ox plants relative to $P H T 1$ in corresponding PHT1ox plants, the expression level of a given PHT may impact root biomass in a dosage-dependent manner by modulating root morphology, which does not directly correlate with arsenic accumulation. Additional evidence for this is seen with the PHTox YCF1ox co-expressors (see below).

Yeast YCF1 was originally identified for its role in vacuolar cadmium sequestration in yeast (Li et al., 1996; Szczypka et al., 1994), and subsequently shown to transport GSH conjugates of not only cadmium, but also mercury (Gueldry et al., 2003) and arsenic (Ghosh et al., 1999). Overexpression of $Y C F 1$ (35S: YCF1) was shown to confer both enhanced resistance to, and accumulation of, lead and cadmium on Arabidopsis plants (Song et al., 2003). Recently, Guo et al. (2012) confirmed increased cadmium accumulation and tolerance of transgenic Arabidopsis overexpressing YCF1 (35S:YCF1), and also showed increased arsenic accumulation and tolerance in these plants. Similarly we demonstrated that overexpression of $Y C F 1$ ( $A 2 p t:: Y C F 1)$ conferred enhanced arsenate resistance (Fig. 4) and accumulation (Fig. 5) to Arabidopsis plants.

By coupling YCF1ox with overexpression of PHT1 or PHT7, we showed further enhancement of arsenate tolerance and accumulation (Fig. 4 and Fig. 5). Although both shoot and root fresh weights of 30-day-old PHTox YCF1ox co-expressors grown on $150 \mu \mathrm{M}$ arsenate were greater than wild type, as well as YCF1ox and PHTox single expressors (Supplementary material, Fig. S1 and Fig. 4), short-term primary root growth assays of young seedlings showed decreases in the PHTox YCF1ox lines compared to wild type (Fig. 6A). This likely reflects a difference in root morphology that correlates with PHTox. Specifically, compared to wild type, all transgenic lines containing a $P H T$-overexpression transgene have relatively shorter primary roots, but greater proliferation of lateral roots, when grown in the presence of arsenate (Supplementary material, Fig. S2). This is consistent with the transgenics overexpressing PHT1 or PHT7 having relatively slower primary root growth in the seedling stage (Fig. 6A), but having greater total root mass at maturity, relative to wild type (Fig. 4C and Supplementary material, Fig. S1). It is possible that increased arsenate acquisition due to $P H T$-overexpression alters root system architecture (RSA), and that the addition of YCF1ox to PHT-overexpressors can compensate for the increased arsenic accumulation, ultimately leading to greater root proliferation, but it cannot reestablish the normal RSA program. This is further supported by comparing the arsenic accumulation and root biomass of all the PHT transgenic lines. In comparing the single PHT-overexpressors, PHT7 expression is 1.75-fold higher than PHT1 expression (Fig. 3), whereas PHT1ox plants have 1.3-fold higher root biomass than PHT7ox plants (Supplementary material, Fig. S1). Similarly, in the PHTox YCF1ox co-overexpression lines, PHT7 expression is 2.6-fold higher than PHT1 (Fig. 3), and the root biomass of PHT1ox YCF1ox plants is 1.3-fold higher than that of PHT7ox YCF1ox plants (Supplementary material, Fig. S1). In contrast, the accumulation of arsenic is not statistically different between PHT1ox and PHT7ox or between PHT1ox YCF1ox and PHT7ox YCF1ox. This suggests that root biomass is more tightly linked to the level of PHT expression than arsenic accumulation. A threshold level of $P H T$ expression appears sufficient for enhanced arsenic accumulation when co-expressed with $Y C F 1$, but root biomass may be more affected by specific PHT expression levels in a way not directly related to arsenate uptake capacity, but perhaps through a more fundamental impact on RSA. 
Depletion of GSH by BSO magnified root arsenate sensitivity in all plants overexpressing PHT1 or PHT7 (Fig. 6A). This highlights the importance of GSH in plant arsenic tolerance. In addition to bringing in more arsenate than wild type, we show that PHTox plants also require a higher intracellular GSH concentration than wild type to support plant growth in the presence of elevated arsenic levels. GSH plays an important role in arsenic detoxification by binding the reduced oxyanion arsenite, to create the arsenite:trisglutathione substrates for ABC transporters such as YCF1 (Ghosh et al., 1999). Hence, the arsenictransporting activity of YCF1 depends on GSH. The PHTox YCF1ox co-expressors were less sensitive to the combination of arsenate and BSO than PHToX alone (Fig. 6A). Also, the PHTox YCF1ox co-expressors contained lower or comparable sulfhydrl levels relative to the PHTox lines under these conditions. Together these results suggest that YCF1 activity is less dependent on de novo GSH synthesis than the ability to tolerate increased arsenate acquisition resulting from $P H T$-overexpression. However, because $Y C F 1$-mediated arsenic transport activity requires GSH, further depletion of GSH by BSO should phenocopy the PHTox root arsenate sensitivity in the PHTox YCF1ox lines. Indeed, we demonstrated that in the presence of higher arsenate and BSO concentrations $(150 \mu \mathrm{M}$ and $500 \mu \mathrm{M}$, respectively) the arsenate sensitivity of PHTox lines was phenocopied in PHTox YCF1ox lines (Supplementary material, Fig. S4).

In conclusion, we demonstrate that Arabidopsis plants overexpressing PHT1 or PHT7 are hypersensitive to arsenate, due to increased arsenic uptake. These plants do not exhibit increased sensitivity to externally supplied arsenite. Co-overexpression of yeast $Y C F 1$ with the PHT1 or PHT7Pi transporters both enhances PHTox-mediated arsenic accumulation, and alleviates $P H T O X$-induced arsenate sensitivity. Taken together, our results support an arsenic transport mechanism in which arsenate uptake is enhanced through Pi transporter overexpression, while arsenic resistance is enhanced through YCF1-mediated vacuolar sequestration.

\section{Supplementary Material}

Refer to Web version on PubMed Central for supplementary material.

\section{Acknowledgments}

We thank Yolanda Lay for assistance with plant care, and anonymous reviewers for helpful comments. This
research was supported by a grant to R.B.M. from the U.S. Department of Energy's Office of Biological and
Environmental Research (DEG0796ER20257), by a NIH National Research Service Award to M.S.L.
(1F32ES015414), and by funds to A.P.S. from Louisiana State University.

\section{References}

Abedin MJ, Feldmann J, Meharg AA. Uptake kinetics of arsenic species in rice plants. Plant Physiol. 2002; 128:1120-1128. [PubMed: 11891266]

Abramoff MD, Magelhaes PJ, Ram SJ. Image Processing with ImageJ. Biophotonics International. 2004; 11:36-42.

Akter KF, Owens G, Davey DE, Naidu R. Arsenic speciation and toxicity in biological systems. Rev Environ Contam Toxicol. 2005; 184:97-149. [PubMed: 15790174]

Aldrich MV, Gardea-Torresdey JL, Peralta-Videa JR, Parsons JG. Uptake and Reduction of Cr(VI) to $\mathrm{Cr}(\mathrm{III})$ by Mesquite (prosopis spp. ): Chromate plant interaction in hydroponics and solid media studied using XAS. Environ Sci Technol. 2003; 37:1859-1864. [PubMed: 12775058]

Berg M, Tran HC, Nguyen TC, Pham HV, Schertenleib R, Giger W. Arsenic contamination of groundwater and drinking water in Vietnam: a human health threat. Environ Sci Technol. 2001; 35:2621-2626. [PubMed: 11452583] 
Bienert GP, Thorsen M, Schüssler MD, Nilsson HR, Wagner A, Tamás MJ, Jahn TP. A subgroup of plant aquaporins facilitate the bi-directional diffusion of $\mathrm{As}(\mathrm{OH})_{3}$ and $\mathrm{Sb}(\mathrm{OH})_{3}$ across membranes. BMC Biology. 2008; 610.1186/1741-7007-1186-1126

Broeks A, Gerrard B, Allikmets R, Dean M, Plasterk RH. Homologues of the human multidrug resistance genes MRP and MDR contribute to heavy metal resistance in the soil nematode Caenorhabditis elegans. Embo J. 1996; 15:6132-6143. [PubMed: 8947035]

Catarecha P, Segura MD, Franco-Zorrilla JM, Garcia-Ponce B, Lanza M, Solano R, Paz-Ares J, Leyva A. A mutant of the Arabidopsis phosphate transporter PHT1;1 displays enhanced arsenic accumulation. Plant Cell. 2007; 19:1123-1133. [PubMed: 17400898]

Clough SJ, Bent AF. Floral dip: a simplified method for Agrobacterium-mediated transformation of Arabidopsis thaliana. Plant J. 1998; 16:735-743. [PubMed: 10069079]

Duker AA, Carranza EJ, Hale M. Arsenic geochemistry and health. Environ Int. 2005; 31:631-641. [PubMed: 15910959]

Durant JL, Ivushkina T, MacLaughlin K, Lukacs H, Gawel J, Senn D, Hemond HF. Elevated levels of arsenic in the sediments of an urban pond: sources, distribution and water quality impacts. Water Res. 2004; 38:2989-3000. [PubMed: 15261536]

Eamens A, Wang MB, Smith NA, Waterhouse PM. RNA silencing in plants: yesterday, today, and tomorrow. Plant Phys. 2008; 147:456-468.

Ellman GL. Tissue sulfhydryl groups. Arch Biochem Biophys. 1959; 82:70-77. [PubMed: 13650640]

Erickson ML, Barnes RJ. Glacial sediment causing regional-scale elevated arsenic in drinking water. Ground Water. 2005; 43:796-805. [PubMed: 16324001]

Ghosh M, Shen J, Rosen BP. Pathways of As(III) detoxification in Saccharomyces cerevisiae. Proc Natl Acad Sci U S A. 1999; 96:5001-5006. [PubMed: 10220408]

Griffith OW, Meister A. Potent and specific inhibition of glutathione synthesis by buthionine sulfoximine (S-n-butyl homocysteine sulfoximine). J Biol Chem. 1979; 254:7558-7560. [PubMed: 38242]

Gueldry O, Lazard M, Delort F, Dauplais M, Grigoras I, Blanquet S, Plateau P. Ycf1p-dependent $\mathrm{Hg}(\mathrm{II})$ detoxification in Saccharomyces cerevisiae. Eur J Biochem. 2003; 270:2486-2496. [PubMed: 12755704]

Guo J, Xu W, Ma M. The assembly of metals chelation by thiols and vacuolar compartmentalization conferred increased tolerance to and accumulation of cadmium and arsenic in transgenic Arabidopsis thaliana. J Hazard Mater. 2012; 199-200:309-313.

Ho SN, Hunt HD, Horton RM, Pullen JK, Pease LR. Site-directed mutagenesis by overlap extension using the polymerase chain reaction. Gene. 1989; 77:51-59. [PubMed: 2744487]

Isayenkov SV, Maathuis FJM. The Arabidopsis thaliana aquaglyceroporin AtNIP7;1 is a pathway for arsenite uptake. FEBS Lett. 2008; 582:1625-1628. [PubMed: 18435919]

Jaquinod M, Villiers F, Kieffer-Jaquinod S, Hugouvieux V, Bruley C, Garin J, Bourguignon J. A proteomics dissection of Arabidopsis thaliana vacuoles isolated from cell culture. Mol Cell Proteomics. 2007; 6:394-412. [PubMed: 17151019]

Jeschke WD, Kirkby EA, Peuke AD, Pate JS, Hartung W. Effects of P deficiency on assimilation and transport of nitrate and phosphate in intact plants of castor bean (Ricinus communis L.). J Exp Bot. 1997; 48:75-91.

Kandasamy M, McKinney E, Meagher R. Functional nonequivalency of actin isovariants in Arabidopsis. Mol Biol Cell. 2002; 13:251-261. [PubMed: 11809837]

Klein M, Burla B, Martinoia E. The multidrug resistance-associated protein (MRP/ABCC) subfamily of ATP-binding cassette transporters in plants. FEBS Lett. 2006; 580:1112-1122. [PubMed: 16375897]

Li Y, Dhankher O, Carreira L, Smith A, Meagher R. The shoot-specific expression of $\lambda$ glutamylcysteine synthetase directs the long-distance transport of thiol-peptides to roots conferring tolerance to mercury and arsenic. Plant Physiol. 2006; 141:288-298. [PubMed: 16581878]

Li Y, Dhankher OP, Carreira L, Lee D, Chen A, Schroeder JI, Balish RS, Meagher RB. Overexpression of phytochelatin synthase in Arabidopsis leads to enhanced arsenic tolerance and cadmium hypersensitivity. Plant Cell Physiol. 2004; 45:1787-1797. [PubMed: 15653797] 
Li ZS, Szczypka M, Lu YP, Thiele DJ, Rea PA. The Yeast Cadmium Factor Protein (YCF1) is a vacuolar glutathione S-conjugate pump. J Biol Chem. 1996; 271:6509-6517. [PubMed: 8626454]

Liu G, Sanchez-Fernandez R, Li ZS, Rea PA. Enhanced multispecificity of Arabidopsis vacuolar multidrug resistance-associated protein-type ATP-binding cassette transporter, AtMRP2. J Biol Chem. 2001; 276:8648-8656. [PubMed: 11115509]

Livak KJ, Schmittgen TD. Analysis of relative gene expression data using real-time quantitative PCR and the 2-ddCT method. Methods. 2001; 25:402-408. [PubMed: 11846609]

Meagher, RB.; Smith, AP.; Pischke, M.; Kim, T.; Dhankher, OP.; Heaton, ACP. Multigene strategies for engineering the phytoremediation of mercury and arsenic. In: Xu, Z.; Li, J.; Xue, Y.; Yang, W., editors. Biotechnology and Sustainable Agriculture 2006 and Beyond. Springer; Netherlands: 2007. p. 49-60.

Meharg AA, Macnair MR. An altered phosphate uptake system in arsenate-tolerant Holcus lanatus L. New Phytol. 1990; 116:29-35.

Meharg AA, Macnair MR. Suppression of the high affinity phosphate uptake system: a mechanism of arsenate tolerance in Holcus lanatus L. J. Exp. Bot. 1992; 43:519-524.

Muchhal US, Pardo JM, Raghothama KG. Phosphate transporters from the higher plant Arabidopsis thaliana. Proc Natl Acad Sci U S A. 1996; 93:10519-10523. [PubMed: 8927627]

Mudge SR, Rae AL, Diatloff E, Smith FW. Expression analysis suggests novel roles for members of the Pht 1 family of phosphate transporters in Arabidopsis. Plant J. 2002; 31:341-353. [PubMed: 12164813]

Murashige T, Skoog F. A revised medium for rapid growth bioassays with tobacco tissue cultures. Physiol Plant. 1962; 15:473-497.

Nagarajan VK, Jain A, Poling MD, Lewis AJ, Raghothama KG, Smith AP. Arabidopsis Pht1;5 mobilizes phosphate between source and sink organs, and influences the interaction between phosphate homeostasis and ethylene signaling. Plant Physiol. 2011; 156:1149-1163. [PubMed: 21628630]

Okano Y, Miki D, Shimamoto K. Small interfering RNA (siRNA) targeting of endogenous promoters induces DNA methylation, but not necessarily gene silencing, in rice. Plant J. 2008; 53:65-77. [PubMed: 17971040]

Papadopoulou B, Roy G, Dey S, Rosen BP, Ouellette M. Contribution of the Leishmania Pglycoprotein-related gene ltpgpA to oxyanion resistance. J Biol Chem. 1994; 269:11980-11986. [PubMed: 7909316]

Patel KS, Shrivas K, Brandt R, Jakubowski N, Corns W, Hoffmann P. Arsenic contamination in water, soil, sediment and rice of central India. Environ Geochem Health. 2005; 27:131-145. [PubMed: 16003581]

Raghothama KG. Phosphate acquisition. Annu Rev Plant Physiol Plant Mol Biol. 1999; 50:665-693. [PubMed: 15012223]

Schat H, Llugany M, Vooijs R, Hartley-Whitaker J, Bleeker PM. The role of phytochelatins in constitutive and adaptive heavy metal tolerances in hyperaccumulator and nonhyperaccumulator metallophytes. J Exp Bot. 2002; 53:2381-2392. [PubMed: 12432030]

Schwartz MS, Benci JL, Selote DS, Sharma AK, Chen AGY, Dang H, Fares H, Vatamaniuk OK. Detoxification of Multiple Heavy Metals by a Half-Molecule ABC Transporter, HMT-1, and Coelomocytes of Caenorhabditis elegans. PLoS One. 2010:5.

Shin H, Shin HS, Dewbre GR, Harrison MJ. Phosphate transport in Arabidopsis: Pht $1 ; 1$ and Pht 1;4 play a major role in phosphate acquisition from both low- and high-phosphate environments. Plant J. 2004; 39:629-642. [PubMed: 15272879]

Smith AH, Hopenhayn-Rich C, Bates MN, Goeden HM, Hertz-Picciotto I, Duggan HM, Wood R, Kosnett MJ, Smith MT. Cancer risks from arsenic in drinking water. Environ Health Perspect. 1992; 97:259-267. [PubMed: 1396465]

Smith AH, Lingas EO, Rahman M. Contamination of drinking-water by arsenic in Bangladesh: a public health emergency. Bull World Health Organ. 2000; 78:1093-1103. [PubMed: 11019458]

Smith E, Smith J, Smith L, Biswas T, Correll R, Naidu R. Arsenic in Australian environment: an overview. J Environ Sci Health A Tox Hazard Subst Environ Eng. 2003; 38:223-239. [PubMed: 12635829] 
Song WY, Sohn EJ, Martinoia E, Lee YJ, Yang YY, Jasinski M, Forestier C, Hwang I, Lee Y. Engineering tolerance and accumulation of lead and cadmium in transgenic plants. Nat Biotechnol. 2003; 21:914-919. [PubMed: 12872132]

Song WY, Park J, Mendoza-Cozatl D, Suter-Grotemeyer M, Shim D, Hortensteiner S, Geisler M, Weder B, Rea P, Rentsch D, Schroder J, Lee Y, Martinoia E. Arsenic tolerance in Arabidopsis is mediated by two ABCC-type phytochelatin transporters. Proc Natl Acad Sci USA. 2010; 107:21187-21192. [PubMed: 21078981]

Srivastava S, Srivastava AK, Suprasanna P, D’Souza SF. Comparative biochemical and transcriptional profiling of two contrasting varieties of Brassica juncea L. in response to arsenic exposure reveals mechanisms of stress perception and tolerance. J Exp Bot. 2009; 60:3419-3431. [PubMed: 19528528]

Szczypka MS, Wemmie JA, Moye-Rowley WS, Thiele DJ. A yeast metal resistance protein similar to human cystic fibrosis transmembrane conductance regulator (CFTR) and multidrug resistanceassociated protein. J Biol Chem. 1994; 269:22853-22857. [PubMed: 7521334]

Vernhet L, Courtois A, Allain N, Payen L, Anger JP, Guillouzo A, Fardel O. Overexpression of the multidrug resistance-associated protein (MRP1) in human heavy metal-selected tumor cells. FEBS Lett. 1999; 443:321-325. [PubMed: 10025956]

Wang J, Zhao FJ, Meharg AA, Raab A, Feldmann J, McGrath SP. Mechanisms of arsenic hyperaccumulation in Pteris vittata Uptake kinetics, interactions with phosphate, and arsenic speciation. Plant Physiol. 2002; 130:1552-1561. [PubMed: 12428020]

Welch AH, Westjohn DB, Helsel DR, Wanty RB. Arsenic in ground water of the United States: occurrence and geochemistry. Ground Water. 2000; 38:589-604.

Wemmie JA, Moye-Rowley WS. Mutational analysis of the Saccharomyces cerevisiae ATP-binding cassette transporter protein Ycflp. Mol Microbiol. 1997; 25:683-694. [PubMed: 9379898]

Xia Y, Liu J. An overview on chronic arsenism via drinking water in PR China. Toxicology. 2004; 198:25-29. [PubMed: 15138026]

Yang XJ, Finnegan PM. Regulation of phosphate starvation responses in higher plants. Ann BotLondon. 2010; 105:513-526.

\section{Appendix A. Supplementary material}

Supplementary material associated with this article can be found in the online version. 


\section{Highlights}

Overexpressing Pht1 family transporters increases arsenate uptake in Arabidopsis.

Overexpressing YCF1 in Arabidopsis increases arsenic tolerance and accumulation.

Co-expressing Pht1 and YCF1 further enhances arsenic tolerance and accumulation. 


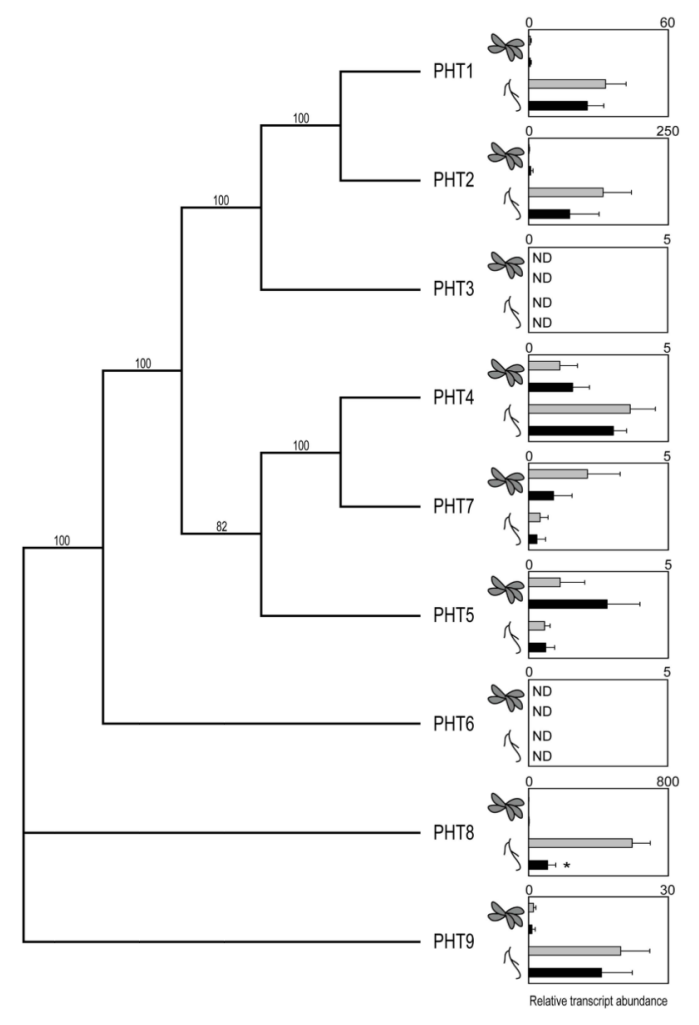

Fig. 1.

Arsenic has a minor impact on the transcript levels of Arabidopsis high-affinity phosphate transporter genes. A phylogenetic tree depicting the relationship of the Arabidopsis PHT family members was prepared in Paup 4.0 using a heuristic method based on distance, after aligning full-length amino acid sequences in ClustalW. The tree was rooted using PHT8 as the out-group, and bootstrapped 1000 times. Bootstrap values are indicated at nodes where applicable. Shoot and root transcript levels for each PHT from 21-day-old seedlings grown in the presence (black bars) or absence (gray bars) of $150 \mu \mathrm{M}$ arsenate are presented relative to the untreated shoot transcript levels for each corresponding PHT. Values are the means of four to six replicates, and error bars indicate standard deviation. Asterisks indicate $P H T$ transcript levels that differ significantly between arsenic-treated and untreated tissues $(\mathrm{p}<$ 0.05). ND, not detected. 


\section{PHT1 transcript abundance}

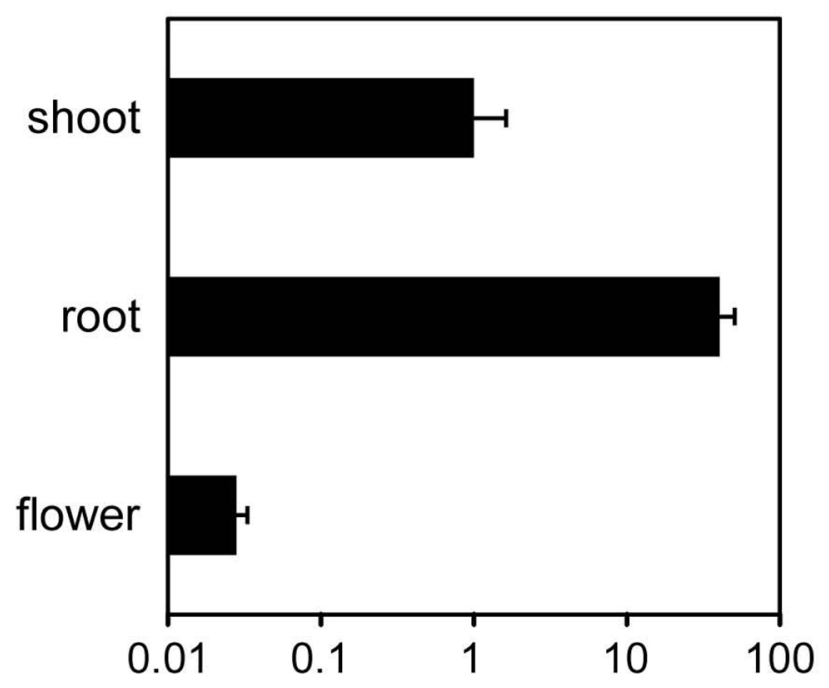

\section{PHT7 transcript abundance}

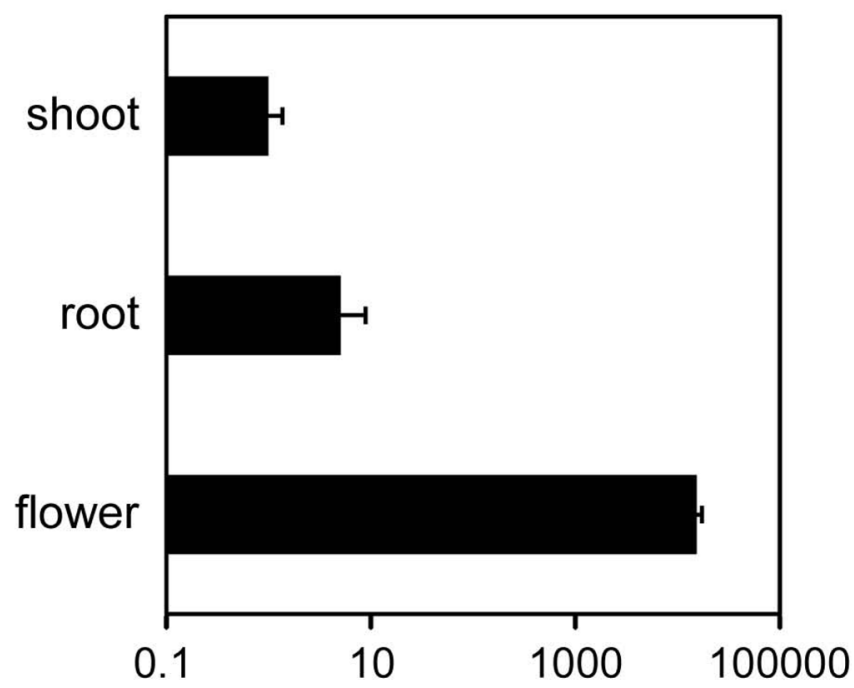

Fig. 2.

Tissue-specificity of PHT1 and PHT7 transcript abundance. PHT1 and PHT7 transcript levels in shoot, root, and flower tissues of wild type Arabidopsis are presented relative to the corresponding transcript level in shoot tissue. Values, displayed on logarithmic plots, are the means of four to six replicates, and error bars indicate standard deviation. 

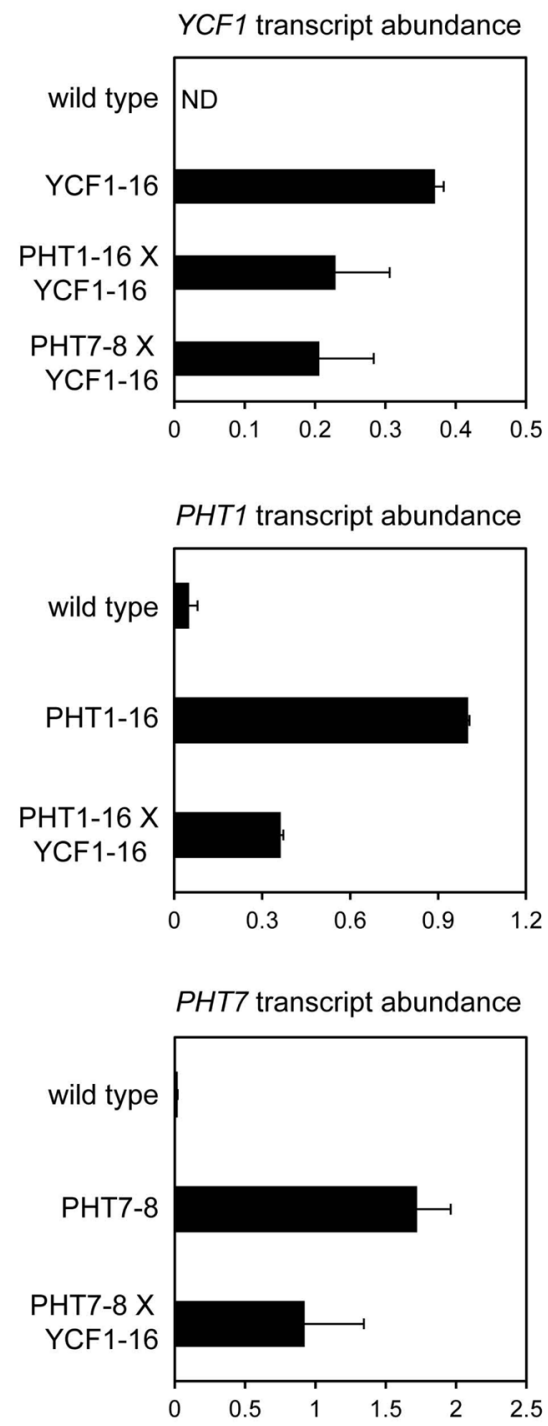

Fig. 3.

Confirmation of transgene overexpression in transgenic seedlings. YCF1, PHT1, and PHT7 transcript levels in whole seedlings of the genotypes indicated are presented relative to the transcript abundance of the ACTIN2 endogenous control gene for each corresponding genotype. Values are the means of four to six replicates, and error bars indicate standard deviation. ND, not detected. 


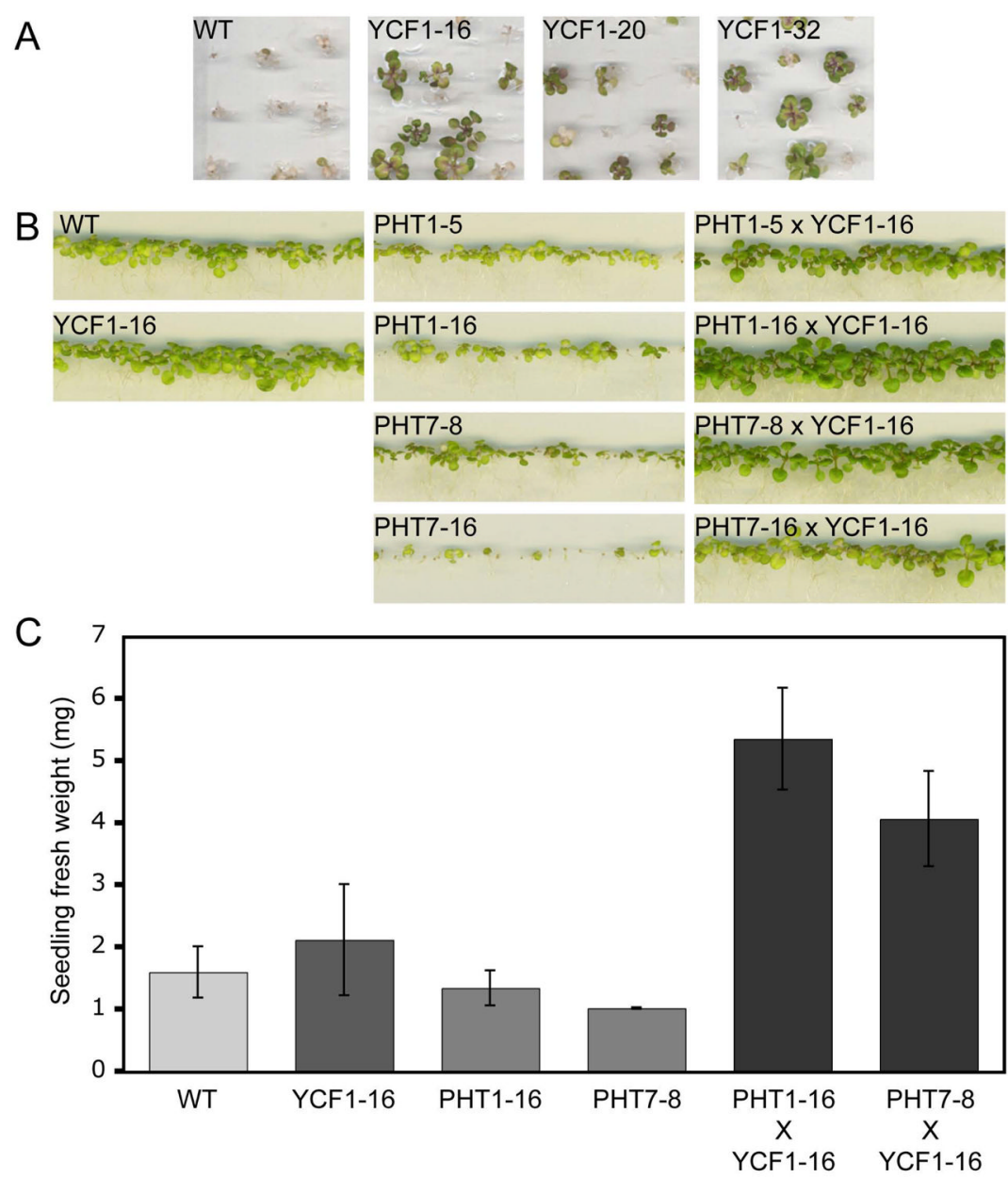

Fig. 4.

Arsenate phenotypes of transgenic Arabidopsis plants overexpressing $P H T$ and/or $Y C F 1$. (A) Wild-type (WT) and transgenic Arabidopsis seedlings segregating for the $Y C F 1$ overexpression transgene were screened for resistance on $200 \mu \mathrm{M}$ sodium arsenate for 30 days. Lines 16, 20, and 32 represent three independent $Y C F 1$-overexpression lines. (B) Wild-type (WT) and homozygous transgenic lines were screened for phenotypes on $150 \mu \mathrm{M}$ sodium arsenate for 30 days. Representative images of two independent PHT1- and PHT7overexpression lines and their corresponding hybrids with $Y C F 1$ (line 16) are shown. (C) Fresh weights of seedlings grown as in panel B were quantified. Error bars indicate standard deviation, $\mathrm{n}>60$. 


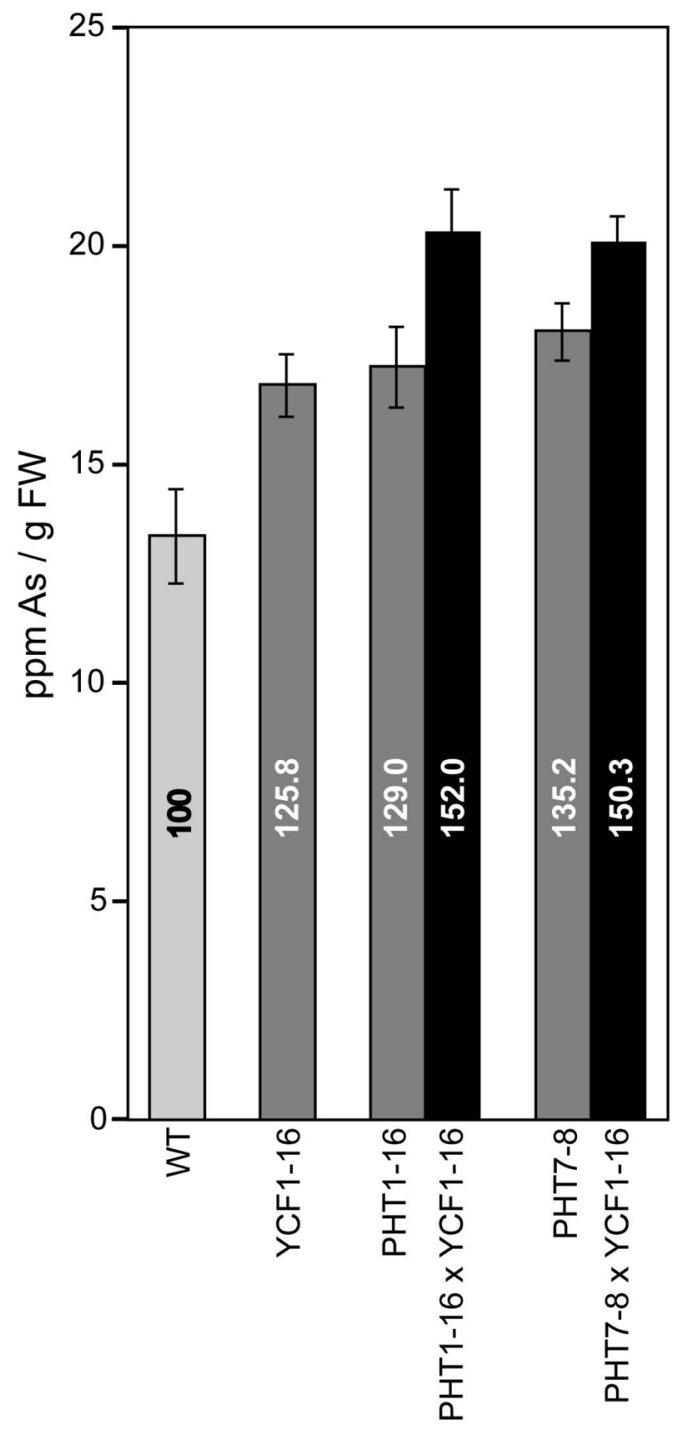

Fig. 5.

Arsenic accumulation in transgenic Arabidopsis plants overexpressing PHT and/or YCF1. Seedling arsenic content of wild-type (WT) and transgenic plants was determined by ICPOES following 21 days of growth on $150 \mu \mathrm{M}$ sodium arsenate. The percent of arsenic accumulation relative to arsenic levels in wild-type plants (100) is noted on each bar. Error bars reflect the standard error, $\mathrm{n}>5$. 


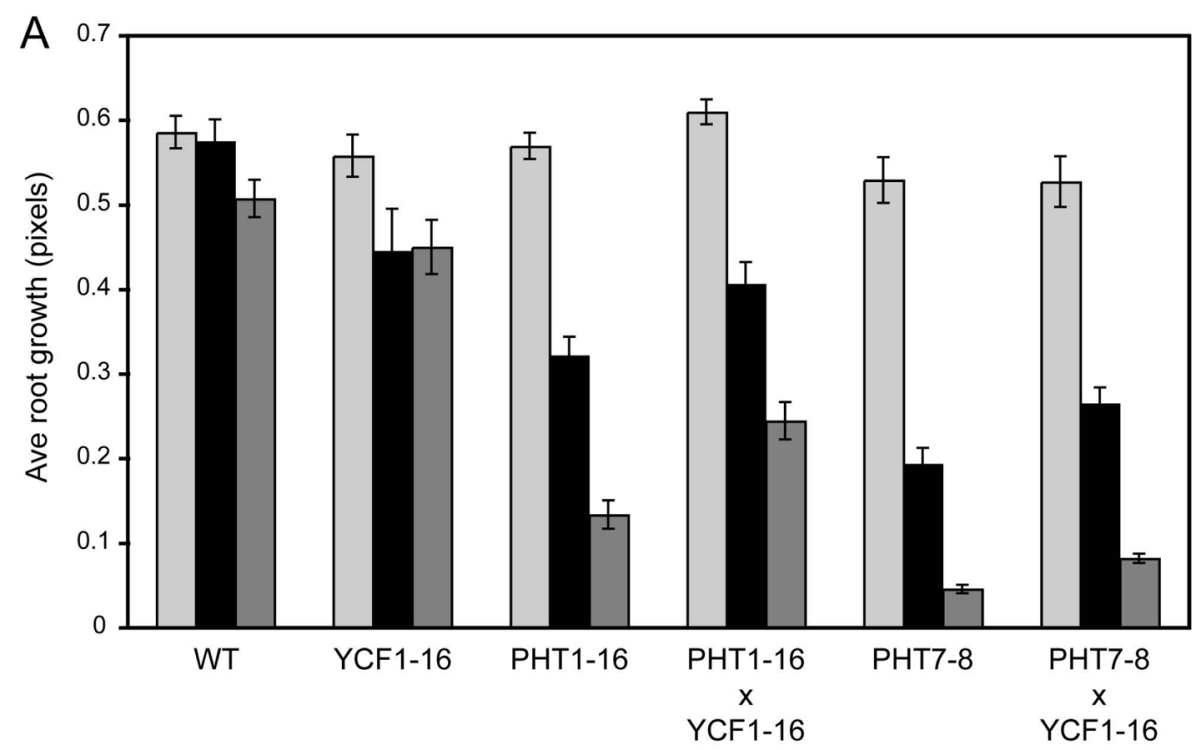

$250 \mu \mathrm{M}$ BSO

$100 \mu \mathrm{M}$ AsV

$250 \mu \mathrm{M} \mathrm{BSO}+100 \mu \mathrm{M} \mathrm{AsV}$

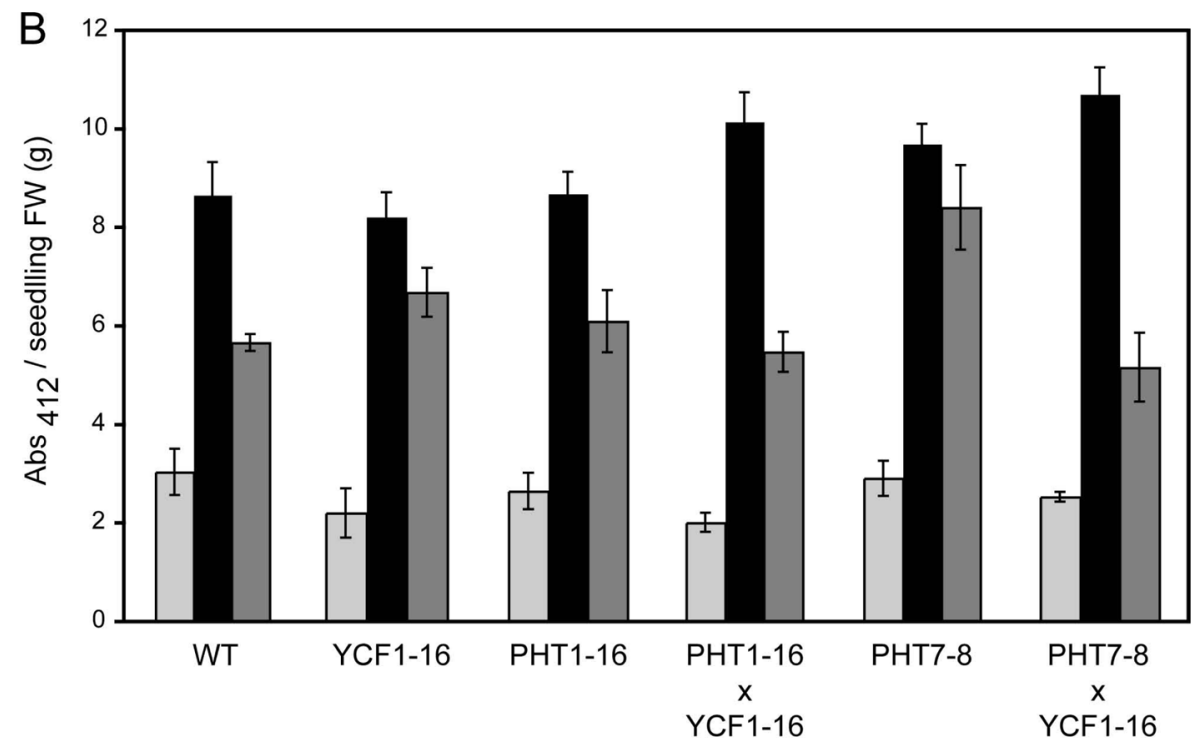

Fig. 6.

The impact of glutathione depletion on arsenate-sensitivity of Arabidopsis plants overexpressing PHT and/or YCF1. Root elongation (A) and seedling sulfhydryl levels (B) were quantified for eight-day old wild-type (WT) and transgenic plants grown for three days in the presence of $250 \mu \mathrm{M}$ BSO, $100 \mu \mathrm{M}$ sodium arsenate (AsV), or both chemicals. Error bars indicate standard error (A) or standard deviation (B), $\mathrm{n} \geq 5$. 